\title{
Carta: Reflexiones sobre posibles razones de una minorización profesional excesiva y propuestas para su reversión
}

\author{
Letter: Reflections on possible reasons for excessive \\ undervaluing of some professions and proposals \\ for its reversal
}

\author{
Lluís M. Anglada
}

Cómo citar este artículo:

Anglada, Lluís M. (2021). “Carta: Reflexiones sobre posibles razones de una minorización profesional excesiva y propuestas para su reversión”. Profesional de la información, v. 30, n. 6, e300604.

https://doi.org/10.3145/epi.2021.nov.04

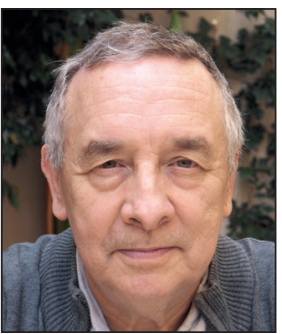

Lluís Anglada

https://orcid.org/0000-0002-6384-4927

Consorci de Serveis Universitaris de

Catalunya (CSUC)

Área de Ciencia Abierta

Gran Capità, 2 (Edifici Nexus)

08034 Barcelona, España

langlada@gmail.com

\section{Resumen}

Algunas profesiones tienen un peso social menor que otras. Su fuerza y poder están por debajo de su peso demográfico o del uso de sus servicios por parte de la población. Nos podemos referir a estas profesiones como profesiones minorizadas. La formada por archiveros, bibliotecas, documentalistas y (quizá) museólogos lo es. Algunas causas de la minorización son generales a la profesión (trabajo funcionarial, auxiliar y mayoritariamente femenino). El artículo no trata éstas, sino las circunstancias que se dan en España que agudizan esta minorización profesional. Dos indicadores de la minorización son: 1) ausencia de la profesión en los foros donde se debaten problemas y soluciones del mundo de la información, y 2) bajo grado de atracción de los estudios entre los jóvenes. En este artículo se intenta definir en qué consiste el valor que aporta la profesión a la sociedad y se analizan los que se han considerado los tres elementos propiamente españoles de minorización, a saber: la secundarización de los estudios, la apolitización de las asociaciones profesionales, y la fragmentación del colectivo. Para cada uno de ellos se dan vías que facilitarían la reversión de esta disminución profesional. Se termina con una reflexión final sobre los impactos negativos de no revertirla.

\section{Palabras clave}

Profesión; Asociaciones profesionales; Defensa profesional; Roles profesionales; Formación; Archiveros; Bibliotecarios; Documentalistas; Museólogos; Archivos; Bibliotecas; Centros de Documentación; Museos; Archivística; Biblioteconomía: Documentación; Ciencia de la información; España.

\footnotetext{
Abstract

Some professions have lower social weight than others, with strength and power below their demographic representation or the use of their services by the population. These can be referred to as minority professions. Archivists, librarians, documentalists, and (perhaps) museologists represent one such example. Some causes of minoritization are general to the profession (e.g., civil servants, auxiliary positions, or work mostly carried out by women). This article does not deal with these, but rather the circumstances in Spain that exacerbate this effect. Two indicators of minority professions are: (1) the absence of the profession from forums where problems and solutions in the world of information are debated, and (2) a low degree of attractiveness of such studies to young people. This article attempts to define the value that a profession contributes to society and analyzes the three elements of such minorization that have been considered particularly Spanish: the secondaryization of studies, the depolitization of professional associations, and the fragmentation of the collective. For each of these, approaches to facilitate the reversal of this professional decline are presented. The work ends with a final reflection on the negative impacts of not reversing this effect.
} 


\section{Keywords}

Profession; Professional associations; Professional defense; Professional roles; Training; Archivists; Librarians; Documentalists; Museologists; Archives; Libraries; Documentation centers; Museums Library science; Documentation; Information science; Spain.

\section{Somos una profesión minorizada}

Si una lengua minorizada es aquella que tiene una presencia social por debajo de su peso demográfico, podemos también hablar de profesiones minorizadas para referirnos a las que tienen una importancia menor que el peso o espacio que ocupan las actividades que realizan sus profesionales. La reciente y aun presente pandemia Covid nos ha dado muchos ejemplos de actividades laborales minorizadas, de ocupaciones a las que no hemos estado dando importancia en condiciones normales pero que han cobrado realce en las presentes circunstancias.

¿La innombrable profesión ${ }^{1}$ formada por archiveros, bibliotecarios, documentalistas y (quizá) museólogos es minorizada? Yo creo que sí, e intentaré razonarlo, para después distinguir entre causas generales y causas propias -o nacionalesde la minorización profesional y, finalmente, proponer medidas para revertir esta situación.

No hay una escala que mida la importancia social de una profesión, pero sin duda hay dos indicadores relevantes de ello:

- su presencia en los foros donde se debaten problemas y soluciones, y

- el grado de atracción que ejercen los estudios correspondientes en los jóvenes.

En los dos suspendemos.

Primero. Una profesión normal -no disminuida-, con un pleno reconocimiento de su función, debería ser una profesión consultada cuando la Administración trata temas que caen dentro de su competencia. Los episodios recientes de la crisis sanitaria y económica provocada por la Covid-19 han dado sobradas muestras de que esto no es así en nuestro caso. Por ejemplo, la profesión ha estado ausente de la reunión que mantuvo el Presidente de la Generalitat de Cataluña con el sector de la cultura a la que asistieron 18 entidades ${ }^{2}$, como ausente estuvo también del acto que reunió a dieciséis representantes de los agentes culturales barceloneses organizado por el diario La vanguardia ${ }^{3}$.

Una profesión normal -no disminuida-, con un pleno reconocimiento de su función, debería ser una profesión consultada cuando la Administración trata temas que caen dentro de su competencia. Los episodios recientes de la crisis sanitaria y económica provocada por la Covid-19 han dado sobradas muestras de que esto no es así en nuestro caso

Gómez-Hernández y Vera-Baceta (2021) analizan la presencia de las bibliotecas públicas españolas en el Fondo europeo de recuperación y en el Plan nacional de competencias digitales y afirman:

"No obstante, no debemos obviar la ausencia de referencia directa a las bibliotecas en el Plan [nacional de educación digital], ya que puede ser entendida como un indicador de que los responsables del impulso de las políticas en pro de la Sociedad de la información y la transformación digital, al igual que parte de la ciudadanía, no cuentan o desconocen algunas de las funciones que pueden desempeñar".

De forma similar Agenjo (2020) comenta:

“... el Gobierno de España acaba de publicar la Agenda España Digital 2025. Y como seguramente ya habrán adivinado esos presuntos lectores, en esa Agenda no hay la más mínima mención a bibliotecas, archivos, museos, ni a centros de documentación..."

De forma menos coyuntural, tampoco se nos incluye entre los profesionales del sector del libro en un estudio de la Fundación Germán Sánchez Ruipérez (2019).

Segundo. Una profesión normal debería ser elegida por los jóvenes cuando seleccionan los estudios a seguir, de forma correlacionada con su importancia e incidencia sociales y su empleabilidad. Pero no ha sido así. Abadal, Ollé y López-Borrull (2021) lo resumen así:

"Los estudios universitarios de Biblioteconomía y Documentación (ByD) en España se empezaron a impartir en 1982 y tuvieron su punto álgido de esplendor a finales de los 90s. A partir del 2000 se inició un descenso en las matriculaciones y se clausuraron algunos programas, iniciándose una crisis en la oferta formativa de la cual aún no se ha podido salir".

Estos autores afirman que los altibajos en la oferta educativa afectan de manera global a todos los países, con un ciclo de máximo esplendor con altas cifras de alumnado, una crisis que comporta el cierre y, finalmente, una cierta recuperación, pero también que

"en el caso de España, como veremos, hemos vivido el apogeo y también la crisis, y ahora mismo aún no se ve claro cómo se puede conseguir la deseada recuperación" (Abadal; Ollé; López-Borrull, 2021). 
Aunque en el momento del inicio de los estudios universitarios se temió que la abundancia de estudiantes matriculados en las facultades de biblioteconomía podría generar mucho desempleo profesional, el mercado laboral fue absorbiendo los titulados bibliotecarios. Es decir, la caída en la demanda en los estudios no es la falta de empleabilidad; en nuestro ámbito la precariedad laboral es un problema más grave que el paro, pero la gente acaba encontrando trabajo y se siente bien en él. Esto debería generar una demanda constante en los estudios de biblioteconomía que no se da. La profesión no atrae a futuros profesionales.

\section{Hernández-Pérez (2019) explica que}

"Ias dos razones principales de la UC3M para suprimir el título de Grado en Información y Documentación (GID) fueron: la alta tasa de abandonos, estudiantes que no finalizaban la carrera, y la caída pronunciada y progresiva del número de estudiantes que demandaban realizar los estudios".

Añadamos aún que las realizaciones profesionales reciben la nota de un excelente por parte de la ciudadanía a quien servimos y que en la sociedad informacional en la que estamos cada vez más metidos (y a pesar de las ayudas mecanizadas que ofrece la tecnología), la función de cuidar o curar la información se ve cada vez más necesaria.

Parece, pues, que nuestra profesión debería ocupar una posición destacada en la escala de consideración social e intelectual, ser considerados, profesionalmente hablando, como indispensables en gran parte de las organizaciones y ocupar posiciones laborales de relevancia en reconocimiento del trabajo bien hecho. Pero ni los éxitos en la gestión ni la necesidad social de la profesión nos han llevado a la ampliación demográfica de la ocupación laboral como tampoco a ocupar posiciones relevantes en la gestión de servicios culturales. Podemos preguntarnos por qué y qué podemos hacer para cambiarlo (si es que creemos que vale la pena cambiarlo).

Los motivos podemos encontrarlos fuera y dentro de nosotros mismos. Las profesiones colisionan entre sí, lo hacen los arquitectos con los aparejadores y también con los ingenieros, los médicos con los farmacéuticos... Las profesiones procuran agrandar los espacios que respectivamente ocupan, y lo hacen con las armas de las competencias y capacidades de cada una, que son los instrumentos que definen las áreas donde se desarrollan. No creo que sea nuestro caso, la nuestra no es una profesión minorizada debido haber perdido espacio respecto de otras profesiones. Si que es una profesión que ha dejado libres demasiados espacios laborales lo que ha hecho que estos fueran ocupados con facilidad por otros profesionales (sobre todo informáticos, pero también técnicos en general).

Hay motivos intrínsecos a la profesión para esta minorización, son motivos que se dan en todas partes donde ésta se ejerce y que se pueden resumir en la poca visibilidad que tienen las profesiones mayoritariamente funcionariales y auxiliares o las consecuencias sociológicas de ser una profesión mayoritariamente femenina. Pero no hablaré de estos motivos, que son generales, pues se manifiestan en la profesión a escala internacional. Están presentes de forma homogénea en todas partes, pero no pesan de forma igual en todos los lugares, porque de ellos no se deriva de forma única la relevancia (o irrelevancia) que nuestra profesión tiene en determinados países.

\section{Sobre las profesiones}

¿Qué define una profesión? Claramente, y, en primer lugar, un conjunto de habilidades, técnicas y de conocimientos. Pero hay un segundo elemento que distingue las profesiones de los oficios y de las ocupaciones. Las profesiones tienen una función social, representan uno de los múltiples valores que componen (o deberían componer) la vida en sociedad, y es por eso que, en determinados casos, la administración delega algunas de sus funciones en las profesiones, o, más concretamente, en las asociaciones profesionales ${ }^{4}$.

Cada profesión tiene sobre la realidad un punto de vista o una mirada que le es propio y que no comparte con las otras profesiones; ante el mismo objeto, el arquitecto ve habitabilidad, el ingeniero seguridad, el economista rentabilidad, el médico salubridad... Cada una persigue un valor -porque perseguirlos todos sería imposible-que será confrontado con los de otras profesiones para finalmente alcanzar un equilibrio entre todos, equilibrio que debería representar la mejor de las soluciones posibles.

La respuesta a un reto (la solución a un problema) debería representar un equilibrio ente puntos de vista distintos. Tal como nos ha mostrado la actual pandemia, las decisiones a tomar no pueden basarse sólo en una mirada o punto de vista -sea esta la salud, sea la economía-. Las cosas en la vida funcionan bien cuando se consigue encontrar un equilibrio entre distintos puntos de vista; esto no responde a una fórmula fija que solo tenemos que aplicar, sino que cambia según el momento y circunstancias. Por esto los constructos sociales funcionan cuando todos los puntos de vista que son relevantes han podido estar presentes y pudieron expresarse y ocupar la posición relativa que les corresponde o que se han ganado. El 'juego' no está en imponer la mirada propia, sino que ésta se tenga en cuenta, que pese.

Una profesión minorizada (que no goza del reconocimiento y/o uso social acorde con su realidad e importancia) no tiene fuerza ni ocasión de exponer su punto de vista, de defenderlo o de imponerlo, y a menudo no lo hace por una autocontención fruto de la minorización misma 
Un oficio no tiene punto de vista o, dicho de otra forma, no aporta valor social, una profesión, sí; las profesiones aportan una determinada mirada sobre la realidad que interesa que se manifieste y exprese en la solución o en el resultado finales. Una profesión minorizada (que no goza del reconocimiento y/o uso social acorde con su realidad e importancia) no tiene fuerza ni ocasión de exponer su punto de vista, de defenderlo o de imponerlo, y a menudo no lo hace por una autocontención fruto de la minorización misma. Cuando una profesión está minorizada su mirada difícilmente pasa a formar parte de la solución y así quien pierde es el conjunto de la sociedad porque el valor que representa la profesión se diluye o pierde.
El punto de vista que representamos es el de usar la información para potenciar las personas y las comunidades, para reforzar sus capacidades y personalidades. La informacionalidad empodera, este es el valor a transmitir, y el éxito de nuestra profesión vendrá de nuestra capacidad de situar la informacionalidad como un valor al lado de la legalidad, la salubridad, la habitabilidad o la seguridad

Es clave, pues, saber lo que es propio de una profesión y lo que no lo es, lo que equivale a preguntarse qué valor representa cada una. En la mayoría de los casos, los valores representados por cada profesión, independientemente de que puedan estar mejor o peor definidos en los estatutos de las asociaciones o colegios respectivos, están muy claros en la opinión común de las personas. Los médicos se ocupan de la salud y los abogados de la justicia. Estas generalizaciones esconden muchos matices, pero tienen la gran virtud de ser operativas; así, cuando alguien tiene un problema sabe a qué profesional dirigirse (otra cosa será la difícil elección de saber cuál es la mejor especialidad dentro de la profesión para cada problema específico).

Por desgracia, este conocimiento intuitivo y generalizado de lo que aportamos no ocurre en nuestro caso. Los 'informacionalistas' no nos hemos sentido a gusto con ningún nombre que nos identificara como profesión y hemos preferido usar los de las especialidades, lo cual ha tenido su reflejo en la organización profesional, que es también fragmentada ${ }^{5}$. Este es un hecho con raíces históricas que lo explican y justifican y que, en mayor o menor grado, se ha dado en todas partes, pero es un hecho que nos debilita porque debilita cualquier imagen profesional (la de las especialidades también) y porqué las fricciones nominalistas consumen considerables energías. La Library Association inglesa cambió su nombre centenario por las incomprensibles siglas de Cilip 6 en un intento de ser vistos por la sociedad como quienes 'represent and champion all information professionals'. Este es un primer paso en la buena dirección, el de mostrar a la sociedad el punto de vista que representamos y para así enseñar que este tiene valor para las personas y para la vida social.

Visto retrospectivamente, creo que nos hemos descrito pensando demasiado en lo que hacemos en relación con la información o la documentación (la facilitamos, la organizamos, la gestionamos...) y menos de lo que hubiéramos debido en lo que aporta la información facilitada, organizada y gestionada. La 'informacionalidad' -si se me permite el palabro- es el resultado de todas estas actividades y significa un estado de vida enriquecida (a nivel personal, formativo y laboral) por el uso de la información. El punto de vista que representamos es el de usar la información para potenciar las personas y las comunidades, para reforzar sus capacidades y personalidades. La informacionalidad empodera, este es el valor a transmitir, y el éxito de nuestra profesión vendrá de nuestra capacidad de situar la informacionalidad como un valor al lado de la legalidad, la salubridad, la habitabilidad o la seguridad.

La digitalización nos debería empujar en esa dirección. En primer lugar, porque si queremos garantizar equidad en esta parte virtual de la vida social (una parte en importancia creciente), la sociedad en general debería estar interesada en que este espacio no esté solo diseñado por profesionales con valores tecnológicos, sino también por profesionales con valores informacionalistas. En segundo lugar, porque la digitalización está unificando las herramientas y las soluciones que usamos desde diferentes especialidades profesionales: los documentos oficiales, los libros de cualquier tipo, la 'literatura gris', las imágenes y cualquier tipo de registros difieren mucho más en el mundo físico que en el digital. En aquel, las características físicas de cada tipo de documento (su unicidad, fragilidad, valor, formato, etc.) imponía tratamientos diferenciados que ahora sus versiones digitales ya no requieren?.

\section{Motivos propios de la minorización de la profesión en España}

He empezado este escrito intentado mostrar que nuestra profesión está seriamente minorizada en España. En un mundo global como en el que vivimos, esto es debido a motivos que se dan en todas partes, a los que se sumarian los propios, de haberlos. Entre aquellos sin duda hay tres: en ser una profesión mayoritariamente funcionarial, en la poca visibilidad general que tienen las profesiones auxiliares, y en el ser una profesión mayoritariamente femenina. Creo que a estos se les suman algunos motivos propios. Mi opinión es que en España la minorización profesional tiene tres componentes que se dan aquí y no forzosamente en otros lugares. Estos serían: la secundarización de los estudios, la apolitización de las asociaciones profesionales, y la fragmentación del colectivo.

Es sabido que la oficialización de los estudios de bibliotecario se hizo en España a partir de una diplomatura (1986). Más tarde a esta oferta se le añadió un segundo ciclo que podía ser cursado tanto por los bibliotecarios diplomados como por toda persona que hubiera terminado un primer ciclo universitario de cualquier carrera. El bienintencionado diseño de este plan perseguía formar técnicos cualificados (diplomados) y, al mismo tiempo, incorporar universitarios 
de las más variadas procedencias que se licenciarían en documentación a partir de sus primeros estudios base en medicina, derecho, informática... Esta no era la única fórmula posible; el modelo anglosajón, tal vez el más generalizado a nivel internacional, forma a los bibliotecarios a través de estudios de postgrado.
Queriendo ser una profesión apolitizada hemos terminado siendo una profesión irrelevante

La elección de este modelo quería tres cosas: dar continuidad a los estudios profesionales impartidos por las escuelas no universitarias de Cataluña y Navarra, dotar a los bibliotecarios titulados medios de un acceso fácil a una licenciatura que les permitiera alcanzar un nivel administrativo asociado al mando, y dar salida a aquellos profesionales que se descubrían documentalistas a posteriori, es decir en el ejercicio de su profesión.

El modelo claramente no ha logrado la atracción de universitarios de otras carreras: los nuevos licenciados han sido mayoritariamente diplomados que alargaban sus estudios. A pesar de que administrativamente la licenciatura en algunos casos se asoció a un requisito para puestos de mando, ésta no se consolidó como la de unos estudios que abrieran ninguna puerta que no la abriera también otra licenciatura universitaria cualquiera. El resultado son unos estudios en horas bajas que no han alimentado a la profesión de personas con la ambición que haría falta para sacar pecho y situar su campo profesional en lugares destacados. Por otro lado, la nota de corte que da acceso a los estudios es baja; aunque esta sea el resultado de una oferta generosa de plazas, el caso es que la imagen que se ofrece es de unos estudios de bajo interés. Así, unos estudios secundarizados han comportado unos profesionales subsumidos.

No todas, pero sí la mayoría de las asociaciones profesionales surgidas en los años 70 y 80 del pasado s. XX fueron profundamente reivindicativas. En muchos casos la misma creación de la asociación respondía más a una voluntad antiminorizadora que la de facilitar el desarrollo y formación de los profesionales. Así fue, por ejemplo, en el caso de la Associació de Bibliotecaris de Catalunya ${ }^{8}$, que nació para acabar con la minorización administrativa de los bibliotecarios que no pertenecían al cuerpo facultativo, o en el de Rebiun de los años 80, que se creó para luchar contra la minorización de las bibliotecas y de sus profesionales dentro de la universidad.

La reorganización de las bibliotecas en la España post franquista fue fruto de asociaciones altamente politizadas, y con ello claramente no me estoy refiriendo a que estuvieran dirigidas por partidos políticos, sino que tenían la intención de influir en la dirección de los asuntos públicos que afectaban a la información y a sus profesionales ${ }^{9}$. Una parte importante de las reivindicaciones se dirigía a que los centros de información fueran gestionados por profesionales, otra a que se dignificaran, modernizaran, ampliaran y/o crearan archivos, bibliotecas y centros de documentación donde hacía falta.

La defensa de la profesión (así lo llamábamos entonces) comportó muchas veces la oposición a decisiones de la administración y enfrentamientos institucionales fuertes y continuados. Puedo entender que, a partir de un momento dado, las asociaciones, ya fuera por cansancio, ya fuera por entender que tendría mejores resultados el apaciguamiento, rehuyeran la confrontación con las administraciones. Una lástima. Este cambio creó la inercia de no hacer proposiciones proactivas en temas sobre los que era apropiado tener un punto de vista profesional. No se han denunciado suficientemente deficiencias del sistema ni aprovechado las oportunidades del continuado cambio tecnológico en nuestro mundo, ni opinado suficientemente a favor de las bibliotecas cuando la crisis económica de 2009, no se ha protestado contra algunas actuaciones más que discutibles de la Administración en determinados concursos ${ }^{10}$... El resultado no ha sido una profesión amable con la que cuenta la administración, sino una profesión a la que no vale la pena convocar cuando se tratan temas 'del sector del libro'. Queriendo ser una profesión apolitizada hemos terminado siendo una profesión irrelevante.

Marquina (2018) relaciona 32 asociaciones profesionales de archiveros, bibliotecarios y documentalistas en España. Son sin duda muchas. La fragmentación es territorial pero también temática. Las hay de documentalistas solos, de estos con bibliotecarios (pero no con archiveros), y de las tres cosas. El área territorial tampoco es determinante ya que existe una autonomía que tiene tres asociaciones y las hay de nivel provincial. En algún caso la asociación se circunscribe a un determinado organismo administrativo en el que se trabaja...

Además de la fragmentación entre archiveros, bibliotecarios y documentalistas, los sub entornos profesionales han hecho hincapié en sus especificidades y diferencias, y así tenemos bibliotecarios escolares y de bibliotecas públicas, de universitarias, documentalistas, archiveros patrimoniales y gestores de la información... Añado que el ejercicio profesional ha quedado muy absorbido por las prácticas de las administraciones públicas, donde de forma muy mayoritaria se ejerce la profesión, cosa que ha acentuado las diferencias entre los del mismo subgrupo pero de diferente diputación o universidad. El resultado de la fragmentación profesional ha sido que nuestra profesionalidad -lo que aportamos a la sociedad- ha quedado diluida y nuestro trabajo desdibujado.

\begin{abstract}
En España la minorización profesional tiene tres componentes que se dan aquí y no forzosamente en otros lugares. Estos serían: la secundarización de los estudios, la apolitización de las asociaciones profesionales y la fragmentación del colectivo
\end{abstract}


Añadamos a la fragmentación asociativa y a la identitaria la de la imagen exterior a partir del nombre que nos damos. Hemos querido ser ingenieros de la información, documentalistas, gestores de información, infonomistas..., pero cuando en nuestras organizaciones las hemos llamado mediatecas, CRAls o mediaLabs hemos tenido que explicar entre paréntesis que se trataba de bibliotecas ${ }^{11}$. Los colectivos minorizados tienen una autoestima baja y en nuestro caso esto se ha reflejado en la insatisfacción respecto un nombre que en el imaginario popular connota la imagen de una profesión en la que no nos sentimos exactamente representados. El estereotipo y la caricatura nos han estigmatizado profesionalmente como una anécdota.

\section{4. ¿Se puede reducir el grado de minorización de la profesión?}

Nuestra profesión, la que tiene como punto de vista propio el de enriquecer la vida personal, laboral y social con información, tiene unos condicionantes internacionales de los que no nos vamos a desprender de forma local. La minorización profesional depende en buena parte de ellos, pero al respecto podemos ser optimistas. Llevamos ya vividas al menos tres décadas de informatización intensiva y hemos constatado que la inmersión intensa en la sociedad informacional no está suponiendo, de momento, la desaparición de los informacionalistas.

En el devenir global en el que ahora todo transcurre, lo que nos pase dependerá de las tendencias generales e internacionales, y estas no son malas. Yo no sé ver que archivos, bibliotecas, centros de documentación y museos vayan camino de la irrelevancia a corto-medio plazo. Es más fácil imaginar un futuro próximo con toda o casi toda la información digitalizada y en red, que el mismo futuro en el que el acceso a la información sea universal de forma automática. Un mundo de información digital en red requiere información tratada, interoperable y preservada ${ }^{12}$ y la interconexión de la información se basa en retos con los que nuestra profesión ya lidió en el mundo de lo impreso.

Estamos bien preparados para tener un rol y éste (nuestra mirada, nuestro punto de vista) es reducir las múltiples barreras al acceso universal a la información: las debidas a los medios tecnológicos para acceder a ella, las de pago o de coste, las de formación y las de oportunidad. Las perspectivas globales e internacionales no son, pues, del todo malas, pero no debemos confiar solo en ellas y sí en cambio añadirles nuestros esfuerzos para cambiar la realidad inmediata.

Centrémonos pues en lo que tenemos más cercano, en lo que he calificado de causas propias o locales de la minorización. Normalmente estamos trabajando en instituciones grandes (gobiernos autonómicos, universidades, organismos de alcance y presencia nacionales) y, por ende, poderosas, y hemos subsumido en ellas nuestro desarrollo profesional, pero hemos de pensar y actuar desde nuestro punto de vista y para nuestros intereses. Dejando de lado que es más fácil diagnosticar que curar, me atrevo a proponer tres caminos para minorizar la minorización:

- formarnos,

- politizarnos, y

- agruparnos.

No hemos tenido suerte con el paso a la universidad de nuestros estudios profesionales ni con su denominación ${ }^{13}$. El modelo de diplomatura más licenciatura de segundo ciclo ha sido demolido por las sucesivas reformas de los grados universitarios. Ni las reformas de los planes de estudio ni la creación de másteres complementarios han conseguido crear un aura de prestigio alrededor de un título universitario. Esto solo podrá conseguirse, creo yo, con la creación de un máster compartido por todas las facultades que imparten estudios profesionales en España, impartido por los profesionales de más prestigio incluyendo un número notable de extranjeros y que seleccione de forma exigente a quienes lo disfrutarán ${ }^{14}$. Hemos de conseguir que la profesión se signifique.

Puedo entender que las generaciones que accedieron a la profesión en los años 90 del siglo pasado consideraran que era el momento de abandonar la actitud muy crítica y beligerante que se tuvo en las décadas anteriores. La ausencia de equipamientos y de presupuestos había sido paliada y podía parecer lo mejor inaugurar una buena relación con la administración basada más en la colaboración que en la confrontación. Pero-como hemos visto- este camino ha resultado estéril desde el punto de vista de los resultados. La profesión debe recuperar el tono reivindicativo y exigente que tuvo. El punto de vista profesional no va a imponerse por sí solo, y menos partiendo de la posición minorizada de la que partimos. Debemos reivindicarnos.

Creo que la fragmentación profesional importante, la que nos hace daño, no es la territorial sino la que separa, por ejemplo, archiveros de bibliotecarios, y a estos de documentalistas, así como los impenetrables muros invisibles que hemos construido entre bibliotecas públicas y universitarias. Hemos creado unas celdas asociativas en las que la igualdad de procedencias nos hace sentir cómodamente bien. Dicho esto, añado que me parece que
Estamos bien preparados para tener un rol y éste (nuestra mirada, nuestro punto de vista) es reducir las múltiples barreras al acceso universal a la información 
la función de Fesabid debería reforzarse. Esto solo podrá hacerse si deja de estar condicionada por las asociaciones que la forman. Si las juntas ejecutivas de la Federación fueran elegidas por sufragio directo de todos los asociados de las entidades que la conforman, esta junta tendría la capacidad de actuar de forma proactiva y sin el freno de mano puesto que es lo que hace ahora ${ }^{15}$. Tenemos que estar y actuar conjuntados.

¿Vale la pena procurar minorizar la minorización sea con estos medios o con otros? Creo que sí y por dos motivos. El primero y más evidente es por nosotros mismos, los profesionales. La salida de la minorización es el camino para un desarrollo profesional mejor y más amplio. Esto incluye -en principio- mejores sueldos, pero también mejor consideración profesional y mejores condiciones de trabajo.

De todas formas, este no es el único motivo ni debería ser el principal. Las profesiones existen por el valor que defienden y el punto de vista que aportan. Pero la particularidad de los valores y las miradas de cada profesión son bienes sociales que benefician al conjunto de la sociedad. La minorización de una profesión lo es también del punto de vista que esta defiende y con ello es todo el mundo quien pierde.

La normalización profesional sería así la mejor garantía de que la sociedad que estamos construyendo sea igualitaria desde el punto de vista del acceso y de la capacidad de uso de la información. Al fin y al cabo, en la sociedad informacional en la que estamos metiéndonos, las desigualdades informacionales van a ser motivo y causa de desigualdades sociales.

\section{Notas}

1. Probablemente una causa de minorización sea la innombrabilidad o falta de nombre para una profesión que hace tiempo y por causas diversas dejó de sentirse representada por la palabra biblioteca. La segunda asociación de bibliotecarios más antigua del mundo -la del Reino Unido-decidió (2002) por este motivo cambiar de nombre y pasar a llamarse Cilip (Chartered Institute of Library and Information Professionals), un nombre incomprensible para el común de los mortales que pronto (2017) tuvo que añadir a su logo la aclaración de que se trataba de la "UKs library and information association". Algo parecido ocurre con las bibliotecas universitarias que han querido darse a conocer con el nombre de CRAI. En este artículo voy a intentar eludir este problema hablando siempre de 'profesión' y englobando en ella a archiveros, bibliotecarios, documentalistas y, si no se ofenden, museólogos.

2. "El Departament de Cultura proposa un paquet de 20 mesures per protegir el sector cultural davant les conseqüències del coronavirus". ICEC, 15 març 2020.

https://icec.gencat.cat/ca/actualitat/noticies/detall/Noticia_Mesures_Govern

3. "Ahora o nunca: la cultura al rescate de la Rambla". La vanguardia, 07 junio 2020 https://www.lavanguardia.com/cultura/20200607/481643474918/barcelona-rambla-cultura.html

4. Debo muchas ideas de este apartado a largas conversaciones con Jordi Ludevid, y a la lectura de su libro Una ciudad de profesiones (My16 EDIT, 2020). ISBN: 8409251361

5. El Cobdc habla de "profesionales de la información", la AAC de los 'archiveros y los gestores de documentos', la Sedic de 'bibliotecarios, documentalistas y otros profesionales de la información', Fesabid, asume como propias las finalidades de sus asociaciones federadas y engloba 'archiveros, bibliotecarios, documentalistas y museólogos y, en general, de todos los profesionales de la información (vid. las webs respectivas).

6. Cilip es el resultado de la fusión, en 2002, de la Library Association -fundada en 1877- con el Institute of Information Scientists -fundado en 1958-

https://en.wikipedia.org/wiki/Chartered_Institute_of_Library_and_Information_Professionals

Una cosa similar hicieron el Col·legi Oficial de Bibliotecaris-Documentalistes de Catalunya (Cobdc) y la Societat Catalana de Documentació i Informació (Socadi) en 1999.

7. La digitalitzación hará que converjamos porqué reduce diferencias entre especialidades profesionales: que lo que era privado y único se ponga en abierto hará que archiveros y museólogos deban acercarse a bibliotecarios y documentalistas; que los objetos digitales deban ser objeto de curación acercará los bibliotecarios-documentalistas a los archiveros-museólogos, etc.

8. Antes de este nombre tuvo que llamarse Asociación de Antiguas Alumnas de la Escuela de Bibliotecología de la Diputación de Barcelona y Associació de Bibliotecàries (sic) de Catalunya.

9. Política es, en acepción 9a de la RAE, la "Actividad del ciudadano cuando interviene en los asuntos públicos con su opinión, con su voto, o de cualquier otro modo".

https://dle.rae.es/pol\%C3\%ADtico

10. Como no hay regla sin excepción, destaco la campaña de Fesabid de "Reivindicación profesional para la Biblioteca Regional de Murcia", a la que se han adherido otras asociaciones (Sedic, que me conste).

https://www.fesabid.org/reivindicacion-biblioteca-regional-murcia 
11. Significativamente, cuando la històrica Llibrary Association del Reino Unido pasó a ser llamarse Cilip tuvo que incorporar al logo que era la "UK's library and information association".

12. El concepto de FAIR, acuñado para los datos de investigación y usado hoy ampliamente, no se refiere más que a esto.

13. Los estudios de periodismo se quedaron con el afortunado nombre de 'Ciencias de la información' que, probablemente sea poco apropiado para ellos mientras que hubiera sido muy conveniente para agrupar de forma neutra nuestras especialidades profesionales.

14. No es exactamente lo mismo, pero la Association of European Research Libraries (Liber) está haciendo algo en esta dirección con los programas de liderazgo, y, concretamente con el de Emerging Leaders.

https://libereurope.eu/working-group/liber-leadership-programmes-working-group

15. Está claro que lo que propongo no supone ninguna modificación del actual panorama asociativo y es, por lo tanto, posible a corto plazo y sin gran esfuerzo.

\section{Referencias}

Abadal, Ernest; Ollé, Candela; López-Borrull, Alexandre (2021). "Library and Information science in Spain: an assessment of 40 years of university studies". Profesional de la información, v. 30, n. 2, e300215.

https://doi.org/10.3145/epi.2021.mar.15

Agenjo, Xavier (2020). "El furgón de cola o los bibliotecarios, archiveros y museólogos, incluso los documentalistas". IweTel, 28 julio.

https://listserv.rediris.es/cgi-bin/wa?A2=IWETEL;dab3a7ce.2007d

Fundación Germán Sánchez Ruipérez (2019). Estudio de la percepción de los profesionales del sector del libro sobre el presente y futuro de la industria: visiones del sector del libro en la era exponencial. Madrid. 104 p. ISBN: 9788489384 989.

https://fundaciongsr.org/wp-content/uploads/2020/01/Visiones-libro-era-exponencial-2.pdf

Gómez-Hernández, José-Antonio; Vera-Baceta, Miguel-Ángel (2021). “Las bibliotecas públicas españolas ante los fondos europeos de recuperación y el Plan nacional de competencias digitales". Anuario ThinkEPI, v. 15, e15b01.

https://doi.org/10.3145/thinkepi.2021.e15b01

Hernández-Pérez, Tony (2019). "Los estudios universitarios de documentación: renovarse para no morir". Clip de Sedic, Revista de la Sociedad Española de Documentación e Información Científica, n. 80 (jul.-dic.).

https://clip.sedic.es/article/los-estudios-universitarios-de-documentacion-renovarse-para-no-morir

Marquina, Julián (2018). "Las principales asociaciones profesionales de archiveros, bibliotecarios y documentalistas en España”. Blog Julián Marquina, 25 septiembre.

https://www.julianmarquina.es/las-principales-asociaciones-profesionales-de-archiveros-bibliotecarios-ydocumentalistas-en-espana

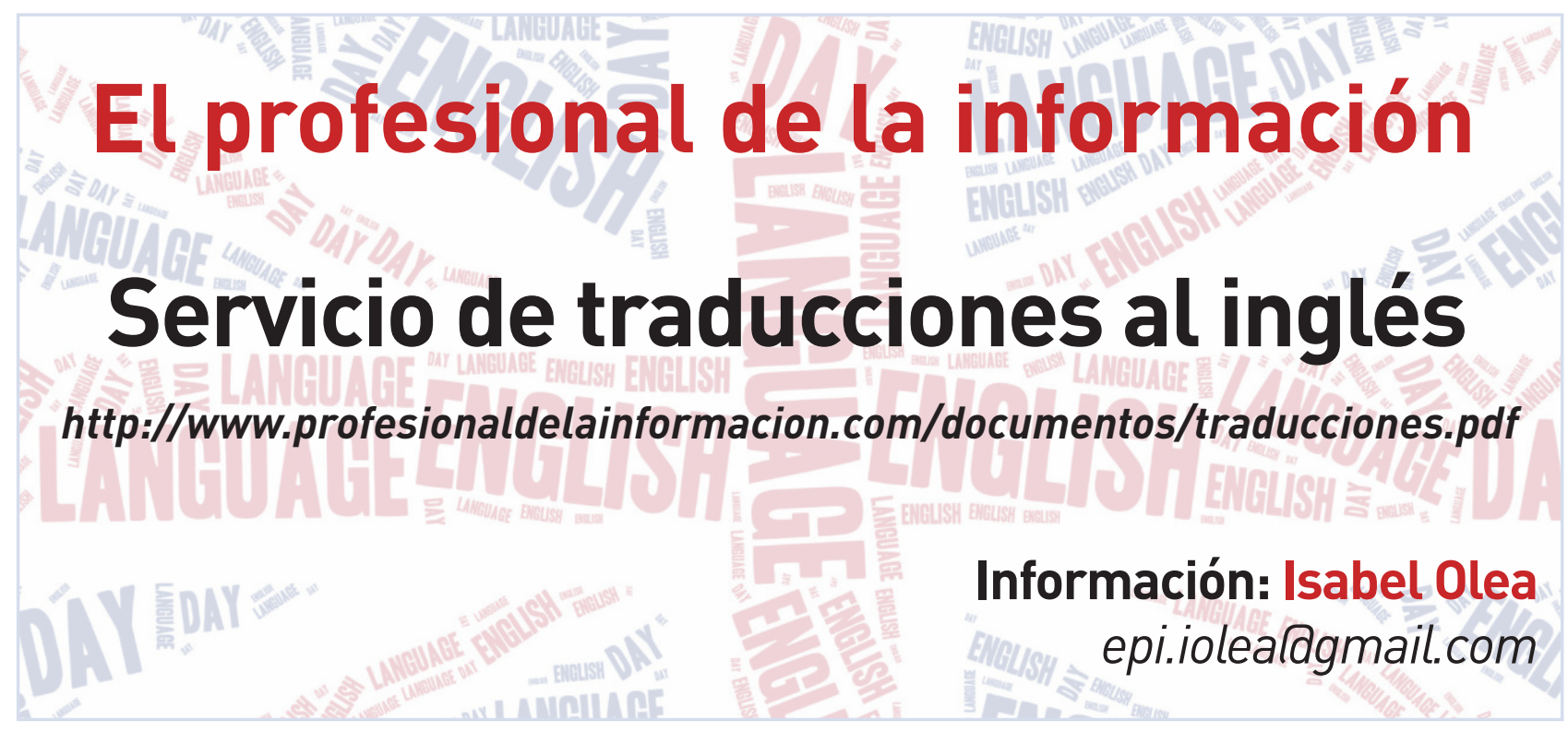

